\title{
La atención a la integración laboral de las personas discapacitadas en la Unión Europea'
}

\section{José Antonio Rodríguez Martín y Juan Delgado Alaminos²}

Palabras clave: Discapacidad, exclusión social, integración, armonización, Unión Europea, empleo.

Key words: Disability, social exclusion, integration, harmonization, European Union, employment.

Mots-clés: Handicap, exclusion sociale, intégration, harmonisation, Union Européenne, emploi.

\section{Introducción: la discapacidad en la Política Social Europea}

La discapacidad es una realidad que presenta múltiples facetas. Según el caso, puede afectar a las personas a lo largo de toda su existencia o alterar el final de sus vidas. En general, los individuos no son iguales ante la discapacidad y el envejecimiento en términos de condiciones de vida y de trabajo. El compromiso asumido por la Unión en favor de sus ciudadanos con discapacidad está asociado a un enfoque

\footnotetext{
' Resultados de un capítulo del Proyecto de investigación: "Evaluación económica de la discapacidad y dependencia en España: distribución geográfica de la necesidad de cuidados paliativos" (2007), financiado por el Ministerio de Trabajo y Asuntos Sociales, la Universidad de Granada y el Departamento de Economía Aplicada de la Universidad de Granada.

${ }^{2}$ Profesores del Departamento de Economía Aplicada, Facultad de Ciencias Económicas y Empresariales, Universidad de Granada.
} 
que no está vinculado con el concepto de asistencia pasiva, sino con los conceptos de integración y de participación activa en la vida económica y social. Así pues, la acción comunitaria se orienta esencialmente hacia el reconocimiento y la protección de los derechos de las personas con discapacidad, si bien las legislaciones nacionales establecen, en la actualidad, las definiciones y los criterios aplicados para determinar una discapacidad, que varían de un Estado miembro a otro.

Las instituciones comunitarias pretenden el establecimiento por parte de los países de una sociedad accesible para todos, reforzando la cooperación y promoviendo la recopilación, el intercambio y la elaboración de datos comparables, de estadísticas y de buenas prácticas, en el marco de la Política Social Europea. Se presta especial atención a los esfuerzos realizados por los Estados miembros para promover la igualdad de oportunidades de las personas con discapacidad, recogiendo contribuciones específicas en las que se presenten sus actuaciones, especialmente en lo que respecta a la integración de la discapacidad en todas las políticas nacionales afectadas.

En este contexto y desde su origen, la Unión ha intentado que la solidaridad constituyese un elemento distintivo del proceso de integración, de manera que uno de los objetivos básicos de los modelos de sociedad y bienestar europeos sería que todas las personas pudieran participar en el progreso económico y social, especialmente los colectivos más desfavorecidos como las personas discapacitadas. Así, no se pretendió construir Europa de un solo golpe, sino poniendo en marcha una solidaridad de verdad entre los países a través de actuaciones concretas como la implantación de una Política Social. En esta línea, se abordó desde un primer momento la unificación económica, instituyendo unas bases similares de desarrollo. Para muchos países no eran suficientes avances en la liberalización del comercio y los pagos, sino que demandaban unos lazos de unión más estrechos y estables que los que se derivaran de una cooperación.

Con este fin, los objetivos de la Política Social definidos en el Tratado de la Comunidad Europea (CE), se inspiraron, en gran medida, en la Carta Social Europea de 1961 y, posteriormente, en la Carta comunitaria de los derechos sociales fundamentales de los trabajadores de 1989, destacando los siguientes objetivos: el fomento del empleo; la mejora de las condiciones de vida y de trabajo; una protección social adecuada; el diálogo social; el desarrollo de los recursos humanos para conseguir un nivel de empleo elevado y duradero; y la lucha contra las exclusiones en el mercado de trabajo de la mujer, las personas discapacitadas, etc.

La Política Social comunitaria centra sus acciones en la formación profesional y en las ayudas a la contratación para los sectores más desfavorecidos y con mayores 
problemas para insertarse en el mercado laboral. Con estas medidas se pretende mejorar la cualificación de los trabajadores para que, como consecuencia, puedan aumentar sus posibilidades para encontrar empleo, elevar su nivel de vida e incrementar la movilidad geográfica, evitar la exclusión social, lograr la inclusión efectiva y la participación íntegra de las personas con discapacidad en la sociedad, entre otros objetivos. En este sentido, la Unión Europea ha suscrito recientemente la Convención de las Naciones Unidas sobre los Derechos de las Personas con Discapacidad, en sintonía con las disposiciones en el ámbito de derechos humanos de la Unión concernientes a esta cuestión ${ }^{3}$.

El objetivo primordial de la Política Social, de acuerdo al preámbulo del Tratado CE, es la mejora de las condiciones de vida y de trabajo de sus pueblos. Los artículos 123 hasta 128 contemplan el órgano comunitario encargado de esta misión, que es el Fondo Social Europeo (FSE) ${ }^{4}$, cuya financiación va destinada a mejorar las posibilidades de empleo de los trabajadores, contribuir a la elevación de su nivel de vida y fomentar la movilidad geográfica y profesional, con especial atención a la inserción laboral de la mujer, las personas discapacitadas, etc.

En esta línea, el Reglamento 1.262/1999 de 21 de junio de 1999, dispone que el FSE tiene por objetivo fundamental la mejora del mercado de trabajo y de los recursos humanos, sosteniendo y completando las acciones de los Estados miembros destinadas al desarrollo del mercado de trabajo y de los recursos humanos en los ámbitos de diversas políticas. Se presta especial atención a los planes de acción nacionales plurianuales para el empleo: el desarrollo y promoción de las políticas activas del mercado de trabajo para combatir y evitar el desempleo, sobre todo el de larga duración; la promoción de la igualdad de oportunidades para todos en el acceso al mercado de trabajo; el fomento y mejora de la formación profesional; la promoción de una mano de obra cualificada, con el refuerzo del capital humano en la investigación, la ciencia y la tecnología; la adopción de medidas específicas para potenciar el acceso y la participación de la mujer en el mercado de trabajo; y mejorar la protección social, con programas destinados a luchar contra la exclusión social de los sectores más desfavorecidos como las personas discapacitadas, fomentando su participación en el empleo.

\footnotetext{
${ }^{3}$ Asamblea General de Naciones Unidas: Sexagésimo segundo período de sesiones; Tema 70 e) del programa: Promoción y protección de los derechos humanos: Convención sobre los derechos de las personas con discapacidad, 22 de enero de 2008 (A/62/654).

${ }^{4}$ El Fondo Social Europeo se creó con el Tratado de Roma, y se consolidó a través de la Decisión 71 /66, de 1 de enero de 1971, y el Reglamento de aplicación de 8 de noviembre de ese año.
} 
Sin embargo, la Política Social y de Empleo no fue prioritaria, ni tuvo relevancia presupuestaria en las primeras etapas de la construcción europea. Por el contrario, en este ámbito las disposiciones se encaminaban hacia el establecimiento de la libertad de circulación de los trabajadores y la libertad de establecimiento, desde la perspectiva del mercado común. Como resultado, hasta la aprobación del Tratado de Ámsterdam en 1999, cuando se implementó el "método abierto de coordinación $(M A C)^{\prime \prime}$, la política social comunitaria no incidía prioritariamente en la lucha contra la discriminación laboral de las personas con discapacidad. Aquel procedimiento se ha traducido en una cooperación entre los Estados miembros en la política de empleo, en la cual la Comisión ejerce una labor de supervisión.

Por lo tanto, en aspectos como la exclusión social, el empleo de las personas discapacitadas, la protección social, entre otros, aunque son competencia de los gobiernos nacionales, hay que subrayar la labor de las instituciones comunitarias en los últimos años respecto a la identificación de los problemas, la elaboración de estadísticas e indicadores o el fomento de las mejores prácticas y la evaluación comparativa entre las medidas adoptadas por los Estados miembros ${ }^{5}$. De este modo, el MAC facilita que las cuestiones relacionadas con la discapacidad se tengan más en cuenta en las materias vinculadas con la Política Social y de Empleo de los países, con la ayuda financiera del FSE.

El FSE cofinancia proyectos que mejoran las posibilidades de empleo de los ciudadanos o que adaptan los recursos humanos al mercado de trabajo, prohibiendo la discriminación por causas de discapacidad u otro motivo. Así, el mayor acceso de las personas con discapacidad al mercado laboral no sólo es uno de los criterios actuales para determinar las actuaciones que pueden financiarse con este Fondo, sino también uno de los principales objetivos de la Política Social Europea. Sin embargo, en la actualidad, en los Estados miembros encontramos diferencias entre las acciones concretas resultantes de las políticas en el campo de las personas discapacitadas, afectadas por diversas experiencias políticas, culturales yeconómicas, que varían de país en país 6 .

\footnotetext{
${ }^{5}$ Según los distintos ámbitos, el MAC supone medidas del denominado "Derecho indicativo", que son más o menos vinculantes para los Estados miembros, pero que no adoptan nunca la forma de directivas, reglamentos o decisiones. Así pues, en el marco de la estrategia de Lisboa, el MAC impone a los Estados miembros la elaboración de planes de reforma nacionales y los transmite a la Comisión.
}

${ }^{6}$ Cfr. Zolkowska, T. (2002). 
Por todo ello, la Política Social Europea busca equiparar las condiciones, mediante mecanismos de coordinación activa, que unan las diversas esferas de la vida social y los factores económicos de los colectivos más débiles, incluyendo disposiciones que benefician a las personas discapacitadas en los diferentes Estados miembros. En este contexto, las políticas comunitarias dirigidas a la búsqueda de la plena integración de las personas con discapacidad, se mueven en muy diferentes campos -salud, educación, formación, accesibilidad, empleo, dependencia-. La integración en este ámbito puede definirse como la inclusión sistemática de las prioridades y necesidades de las personas con discapacidad en todas las políticas y medidas generales de la Unión, con el fin de fomentar la igualdad de oportunidades de este colectivo.

Como quiera que el presente trabajo no puede abordar todas las intervenciones, nuestro objetivo es analizar un aspecto de gran relevancia, la integración laboral de las personas discapacitadas, en el marco de la Política Social Europea, para lo que es preciso repasar la evolución normativa en esta materia en los últimos años. Con este fin, los principales avances alcanzados en la Unión en este terreno se repasan secuencialmente en los puntos siguientes de nuestro trabajo, desde la entrada en vigor del Tratado de Ámsterdam en 1999, que incorporó el objetivo de hacer del empleo y de los derechos de los ciudadanos, con especial atención a los grupos más desfavorecidos, uno de los ejes de la Unión Europea, hasta la actualidad.

\section{Nuevas bases para la legislación comunitaria en la inclusión laboral y protección social en la Unión: el Tratado de Amster- dam (1999)}

En la Unión Europea, los países coordinan sus políticas con el fin de que todo ciudadano tenga acceso a prestaciones sociales que aseguren pensiones y atención sanitaria de calidad a un coste sostenible; que potencien la inclusión social y luchen contra la pobreza; que impulsen una acción global contra todas las formas de discriminación; y que apoyen medidas para favorecer la integración de los discapacitados, etc. En concreto, y en lo relativo a la no discriminación, el fin específico del artículo 12 (antiguo artículo 6) del Tratado CE ha sido, históricamente, el de evitar cualquier discriminación por motivo de la nacionalidad. No obstante, esta disposición no era suficiente para dotar una herramienta eficaz en la lucha contra otro tipo de discriminaciones como el de las personas con discapacidad en el mercado laboral. 
Aunque la Política Social tiene un reconocimiento explícito en el Tratado Constitutivo de la Unión, los esfuerzos para formar un espacio social europeo no fructificaron en las primeras etapas del proceso de integración. Además, el Tratado de la Unión Europea de Maastricht, que se firmó el 10 de febrero de 1992, aunque abrió una etapa decisiva en el proyecto de integración, dado que fijó las fases hacia la Unión Económica y Monetaria (UEM), no contempló ningún avance en la integración laboral de los segmentos de la población más desfavorecidos como los discapacitados.

Por esa razón, el Tratado de Ámsterdam (artículo 13) ${ }^{7}$, amplió el principio de no discriminación comentado, contemplando que sin perjuicio de las demás disposiciones del presente Tratado y dentro de los límites de las competencias atribuidas a la Comunidad por el mismo, el Consejo, por unanimidad, a propuesta de la Comisión y previa consulta al Parlamento Europeo, podrá adoptar acciones adecuadas para luchar contra la discriminación por motivos de sexo, de origen racial o étnico, religión o convicciones, discapacidad, edad u orientación sexual. Esta disposición se complementa con otros artículos, que potencian aún más la lucha contra todo tipo de discriminaciones, especialmente en lo que respecta a las personas con discapacidad (apartado $n^{\circ} 22$ de la Declaración del Acta final del Tratado de Ámsterdam, que hace referencia al artículo 95 del Tratado CE, con una Declaración relativa a esta materia): Las instituciones comunitarias, al elaborar medidas con arreglo al artículo $100 \mathrm{~A}$ del Tratado constitutivo de la Comunidad Europea, deberán tener en cuenta las necesidades de las personas discapacitadas.

En este marco normativo, las instituciones comunitarias consideran a la discriminación como un obstáculo a la cohesión económica y social de la Unión Europea, por lo que la Comisión considera intolerable cuando se refiere, especialmente, a personas que necesitan asistencia, como las personas con discapacidad o los ancianos. En general, y siguiendo la doctrina del Tribunal de Justicia de las Comunidades Europeas, se considera discriminación a la aplicación de normas diferentes en situaciones comparables o la aplicación de una misma norma a situaciones diferentes.

\footnotetext{
7 En lo referente a los derechos sociales, la gran novedad la constituye que el Reino Unido, tras la llegada al poder del laborista Tony Blair, se ha adherido al Acuerdo Social que iba anexo al Tratado de Maastricht. Este protocolo ha sido incluido en el Tratado de Ámsterdam y, por él, los estados miembros se comprometen a respetar los derechos sociales recogidos en la Carta Comunitaria de Derechos Sociales, aprobada en 1989, conocida normalmente como Carta Social. La Unión Europea puede en adelante actuar en los ámbitos de salud y seguridad de los trabajadores, las condiciones de trabajo, la integración de las personas excluidas en el mercado laboral o en la igualdad de trato entre hombres y mujeres.
} 
Hay que tener en cuenta, que los distintos motivos de discriminación son bastante variados, ya que pueden estar basados en la raza o el origen étnico, la discapacidad, la edad, la orientación sexual, el sexo, la religión o incluso las convicciones, afectando de este modo todos los aspectos de la vida corriente. Así pues, pueden encontrarse múltiples casos como el de evitar la discriminación en la inserción profesional de las personas con discapacidad, objetivo que se reforzó ulteriormente en el Tratado de Niza en 2001. Este Tratado, aún en vigor, pretendía, en general, reformar las instituciones comunitarias para que funcionara de forma efectiva una Unión de veinticinco miembros, tras la ampliación hacia el Centro y el Este de Europa, pero sin olvidar la relevancia de los aspectos sociales en el proceso de integración.

En concreto, el Tratado de Niza incorporó la posibilidad de que el Consejo adopte medidas comunitarias de estímulo para apoyar las acciones de los Estados miembros destinadas a combatir todo tipo de discriminación por mayoría cualificada, con especial atención a las personas discapacitadas. Esta prioridad se trasladó en 2004 a la estrategia de igualdad y no discriminación en los Estados miembros, plasmada en el Libro Verde en la Unión ampliada ${ }^{8}$, que abordamos a continuación.

\section{LibroVerde (2004): igualdad y no discriminación en la Unión ampliada}

En la actualidad, las personas con discapacidad suponen, aproximadamente y como mínimo, el $15 \%$ de la población total de la Unión Europea en edad laboral. Por otro lado, a principios del siglo XXI, la Unión se enfrenta a cambios demográficos sin precedentes, que tendrán importantes repercusiones en el conjunto de la sociedad, con una correlación entre el creciente envejecimiento de la sociedad europea, la mejora del sistema sanitario y una mayor presencia de la discapacidad.

Ante estos importantes retos, en el Libro Verde, la Comisión Europea esbozó en 2004 los avances registrados contra los diferentes tipos de discriminación, con especial atención a las personas con una discapacidad. Además, planteó actuaciones futuras para potenciar su implicación en este ámbito en los próximos años. En el documento se subrayaba que en otros ámbitos como el de la igualdad de sexo, la Unión adoptó numerosas medidas a partir de 1975, pero que no se avanzaba

${ }^{8}$ Libro Verde - Igualdad y no discriminación en la Unión Europea ampliada [COM (2004) 379 final - no publicado en el Diario Oficial]. 
apenas en la lucha contra otros tipos de discriminación. De ahí la importancia que el Libro Verde le atribuye a la aprobación del artículo 13 mencionado del Tratado de Ámsterdam en 1999, que permite a las instituciones comunitarias tomar medidas contra la discriminación sustentada en nuevos motivos como una discapacidad.

Como resultado, en virtud de las nuevas disposiciones introducidas, la Comisión pudo presentar en noviembre de 1999 una comunicación global contra las discriminaciones (COM (1999) 564 final) $)^{9}$, tras una amplia consulta con los Estados miembros. Esta propuesta se tradujo en un gran avance en la legislación comunitaria sobre discriminación, planteando el marco general de actuación de la Unión, de tal modo que vino acompañada de varias directivas, decisiones y reglamentos, destinados a garantizar la igualdad de trato a las personas con discapacidad, que se analizan más adelante.

Sin embargo, hay que resaltar que no es fácil medir regularmente la eficacia de la legislación y las políticas antidiscriminatorias en los diferentes países, debido, principalmente, a la ausencia de datos actualizados y comparables. De hecho, los Estados miembros ya prohíben la discriminación en el mercado de trabajo, si bien sus legislaciones al respecto son desiguales respecto al alcance, el contenido y la fuerza de ejecución. En este sentido, cabe subrayar el tiempo y los esfuerzos que conlleva la aplicación y armonización de cualquier medida en el campo de la lucha contra la discriminación como, por ejemplo, la Recomendación 98/376/CE del Consejo, de 4 de junio de 1998, sobre la creación de una tarjeta de estacionamiento para personas con discapacidad ${ }^{10}$. Esta disposición pretendía, en última instancia, armonizar la forma de los permisos de estacionamiento para personas con discapacidad, lo que favorecería el desplazamiento a sus puestos de trabajo, por lo que se demandaba el reconocimiento mutuo de dichos permisos por los Estados miembros de la Unión, objetivo que no ha resultado fácil alcanzar, pese a los esfuerzos realizados por las diferentes instituciones.

En particular y desde una óptica legal, para garantizar a los ciudadanos europeos una mayor protección, y en el marco de la regulación de la dependencia en el Derecho interno de la Unión Europea, en 2000 el Consejo adoptó una Directiva relativa a la igualdad en materia de empleo (Directiva 2000/78/CE), así como el acoso y las medidas discriminatorias (Directiva 2002/73/CE). Como conse-

\footnotetext{
${ }^{9}$ COM (1999) 564 final. Comunicación de la Comisión sobre algunas medidas comunitarias de lucha contra la discriminación.
}

${ }^{10}$ [Diario Oficial L 167 de 12.6.1998]. 
cuencia, se ha obligado a todos los Estados miembros a introducir paulatinamente modificaciones en sus legislaciones antidiscriminatorias nacionales, con el objeto de cumplir la normativa comunitaria en temas de ocupación de personas con discapacidad.

Paralelamente, todos los empresarios deberían realizar los ajustes razonables - tomar las medidas adecuadas para permitir el acceso y el mantenimiento del empleo a las personas con discapacidad. Esta Directiva pretende instaurar un marco general de requisitos mínimos comunes, esto es, contiene una cláusula "no regresiva" para los Estados que aplican una legislación con un nivel de protección más elevado que el que prevé la propia Directiva. Por otra parte, hay que tener en cuenta que se obliga al Estado miembro destinatario en cuanto al resultado que debe conseguirse, pero son las autoridades nacionales quienes eligen la forma y los medios para ello.

La Directiva se aplica, incluidos los organismos públicos, a las condiciones de acceso de los sectores más desfavorecidos como las personas con discapacidad al conjunto de los empleos y las actividades por cuenta propia y las condiciones de promoción; la formación profesional; las condiciones de empleo y de ocupación (incluidas las de despido y remuneración); la inscripción y participación en una organización patronal, sindical o en cualquier organización profesional. Asimismo, esta disposición va dirigida tanto a la discriminación directa (diferencia de trato basada en una característica determinada) como a la indirecta (disposición, criterio o práctica aparentemente neutros, pero que pueden tener efectos desfavorables para una o varias personas determinadas o incluso producir una incitación a la discriminación).

En 2000, se adoptó también una Decisión, que estableció por seis años (2001-2006), un programa comunitario para apoyar a los Estados miembros en el desarrollo de políticas contra la discriminación gracias a un intercambio de informaciones y buenas prácticas en materia legislativa y no legislativa ${ }^{11}$. El objetivo era conocer mejor los problemas existentes en cada país y sensibilizar mejor a los protagonistas que pueden luchar contra las discriminaciones en Europa y reforzar la cooperación con otras instancias. Este programa estuvo dotado con un presupuesto de 98,4 millones de euros. Singularmente, se destinó una gran proporción de los recursos humanos y financieros del programa a proyectos transnacionales, que reunían a

\footnotetext{
${ }^{11}$ La elaboración del programa contra la discriminación de la Unión, se benefició de contribuciones de toda una serie de socios, entre ellos las autoridades nacionales, el Parlamento Europeo, los interlocutores sociales y las ONG, las autoridades regionales y locales, el Comité de las Regiones, expertos universitarios y el Observatorio Europeo de los Fenómenos Racistas y Xenófobos.
} 
distintos grupos y organizaciones como al Foro Europeo de Personas con Discapacidades (FEPH) ${ }^{12}$, así como a una serie de redes más pequeñas activas en el ámbito de las minusvalías.

Posteriormente, se aprobó en 2002 un Reglamento sobre ayudas al empleo, destinado a facilitar las iniciativas de los Estados miembros orientadas a la creación de nuevos puestos de trabajo. En virtud de esta norma, los gobiernos nacionales pueden conceder ayudas a la generación de empleo y a la contratación de trabajadores discapacitados sin contar con la autorización previa de la Comisión. Tratándose de parados de larga duración y de otros trabajadores desfavorecidos, se pueden hacer cargo por un año del $50 \%$ de los costes salariales y las contribuciones obligatorias a la Seguridad Social. En el caso de los empleados discapacitados, es posible asumir hasta el $60 \%$ de estos costes. Este nuevo Reglamento no prejuzga otros tipos de ayudas, pero tales medidas deberán ser notificadas individualmente a la Comisión, que las examinará caso por caso.

Además, la Comisión Europea adoptó en octubre de 2003 un plan específico de acción europeo en este ámbito. Este Programa enuncia una serie de iniciativas destinadas a promover el acceso de las personas discapacitadas al empleo, la educación y la formación permanente, las nuevas tecnologías y los edificios, en el marco del principio internacional de no discriminación, recogido anteriormente en la Carta de los Derechos Fundamentales de la Unión. En este documento la Unión reafirmó, en diciembre de 2000, con motivo de la Conferencia Intergubernamental de Niza, el compromiso con el principio de no discriminación. Su artículo 21 prohíbe la discriminación basada en los seis motivos de discriminación mencionados en el artículo 13 del Tratado CE, incluido el de una persona con discapacidad.

El derecho a la no discriminación es reconocido, asimismo, por los principales organismos multilaterales como la Declaración Universal de los Derechos Humanos, el Pacto sobre derechos económicos, sociales y culturales de la ONU ${ }^{13}$, el Convenio internacional sobre la eliminación de todas las formas de discriminación racial o el Convenio $\mathrm{n}^{\circ} 111$ de la Organización Internacional del Trabajo (OIT).

\footnotetext{
${ }^{12}$ El FEPH es una organización central europea que representa a 70 organizaciones europeas no gubernamentales, 17 consejos nacionales y 37 millones de ciudadanos discapacitados de todos los países de la Unión y de la Asociación Económica Europea. Su principal misión es la de hacer avanzar los derechos humanos de los discapacitados y promover la igualdad en las instituciones de la Unión y en los Estados miembros, con la cooperación cada vez mayor y más activa de la Comisión.
}

${ }^{13}$ Comunicación de la Comisión de 2003 Hacia un instrumento jurídicamente vinculante de las Naciones Unidas para promover y proteger los derechos y la dignidad de las personas con discapacidad. 
Las disposiciones relativas a la no discriminación contenidas en el Convenio europeo de protección de los derechos humanos y las libertades fundamentales fueron reforzadas recientemente por la entrada en vigor, el 1 de abril de 2005, de un nuevo protocolo a dicho convenio, que instaura un derecho autónomo a la igualdad de trato. También, la recientemente aprobada Carta de los Derechos Fundamentales de la Unión Europea (2007/C/303/01), de 12 de diciembre de 2007, ha dispuesto -en su artículo 21- la prohibición expresa de todo tipo de discriminación. Así, la legislación de la Unión contra la discriminación, se puede considerar como una de las más elaboradas del mundo. Este hecho se tradujo, incluso, en la instauración del Año Europeo de las personas con discapacidad en 2003, con la celebración de una sesión en el Parlamento Europeo con discapacitados de toda la Unión, con el objetivo de discutir que mejoras eran necesarias en el ámbito comunitario en esta materia.

En general, se pretende avanzar progresivamente en el reconocimiento y la protección de los derechos de este colectivo en la sociedad. En la actualidad, la mayor parte de estos planteamientos se recogen en la estrategia europea de empleo y la Agenda Social, en la que se reconoce que el acceso al mercado laboral es determinante para la integración económica y social de las personas con discapacidad.

\section{La estrategia europea de empleo (2005-2008) y la Agenda Social (2005-20 I 0)}

La vigente Estrategia Europea de Empleo (EEE), orientada a la creación de puestos de trabajo, y la Agenda Social (2005-2010)14, destinada a garantizar que las ventajas del crecimiento alcancen a toda la sociedad, constituyen las bases de la política social y de empleo de la Unión en los próximos años. El Fondo Social y el programa comunitario para el empleo y la solidaridad social (PROGRESS) apoyan económicamente esos objetivos.

De este modo, todos los Estados miembros elaboran de común acuerdo unas directrices de empleo plurianuales e individuales para sus políticas en esta materia. Estos criterios constituyen la piedra angular de los programas nacionales de reforma y

\footnotetext{
${ }^{14}$ Sobre la base de los objetivos de la Agenda Social (2005-2010), la Comisión presentó la Comunicación de "Situación de las personas con discapacidad en la Unión Europea ampliada: el plan de acción europeo 2006-2007".
} 
el componente en materia de empleo del Programa de Lisboa de la Unión ${ }^{15}$, que incluye entre sus prioridades el avance de los niveles comparativamente bajos de la participación en el mercado de trabajo de las personas europeas con discapacidad. En este marco, algunos gobiernos nacionales comenzaron a desarrollar una estrategia que combina las medidas destinadas a promover la integración de grupos desfavorecidos y actuaciones de lucha contra las actitudes, comportamientos y prácticas discriminatorias.

Como resultado, al seguir las orientaciones fijadas por la EEE, los Estados miembros están obligados a informar anualmente a la Comisión Europea en cuanto a las iniciativas nacionales en materia de empleo, incluido el destinado para personas con discapacidad. Las directrices tienen en cuenta la necesidad de aplicar políticas destinadas a lograr el pleno empleo, mejorar la calidad y la productividad en el trabajo y reforzar la cohesión social y territorial, objetivos que se complementan y realzan con las medidas derivadas de la Agenda social de la Unión (2005-2010).

La Agenda abarca políticas destinadas especialmente a la lucha contra la pobreza y el fomento de la igualdad de oportunidades para todos, incluidos los segmentos de la población más desfavorecidos por el mercado laboral como las personas discapacitadas. Estas políticas se elaboran en colaboración con las autoridades de todos los niveles, impulsando a los países a abordar las cuestiones sociales y de empleo, que vayan surgiendo a medida que envejece la población, fomentar la igualdad de oportunidades y erradicar la discriminación.

En este contexto, se instaura en 2007 el Año Europeo de la lgualdad de Oportunidades para todos, que puso de manifiesto la importancia de la presencia de la discapacidad en las políticas de la Unión. Así, los temas vinculados con esta materia están ya incluidos en diferentes programas de financiación, entre los que sobresalen estudios estadísticos, Programa marco de Investigación de la Comunidad o iniciativas de educación. Para el cumplimiento de estas prioridades, el FSE dispone de 308.000 millones de euros de 2007 a 2013 para, entre otras muchas actuaciones, luchar contra la discriminación, facilitar el acceso de los discapacitados al mercado laboral, mejorar los sistemas de

\footnotetext{
${ }^{15}$ En el Consejo Europeo de Lisboa de marzo de 2000, los Jefes de Estado o de Gobierno europeos reconocieron que Europa debía convertirse en una economía integradora, dinámica y basada en el conocimiento, impulsando un crecimiento económico acelerado y sostenido. De hecho, fijaron un nuevo objetivo de la Unión: convertirse en la sociedad basada en el conocimiento más competitiva del mundo en 2010.
} 
educación y formación y desarrollar la facultad institucional en las regiones más desfavorecidas ${ }^{16}$.

En esta línea y en el ámbito de la igualdad de oportunidades en el mercado de trabajo, la Comisión Europea fomenta la responsabilidad social de las empresas, promoviendo la idea de que los aspectos sociales y ambientales deben formar parte integrante de las estrategias empresariales ${ }^{17}$. Cabe mencionar el caso de algunos países como Dinamarca, que ha establecido un plan de acción propio en el ámbito de las personas con discapacidad y ha previsto construir en los próximos años nuevas viviendas adaptadas totalmente a las personas con discapacidad; o el ejemplo de Alemania, que ha puesto en práctica la nueva ley sobre la igualdad de trato de los discapacitados, paralelamente al desarrollo de la legislación marco anterior. Así, varios países europeos disponen de una legislación contra la discriminación, que ha sido útil para eliminar paulatinamente las barreras como las arquitectónicas, pero también las de comportamiento, que las personas con discapacidad encuentran en la sociedad. En este sentido, el Reino Unido dispone de una legislación contra la discriminación muy avanzada, y en los Países Bajos y España, entre otros, se están tomando medidas para promover una legislación más eficaz contra la discriminación.

En este contexto, hay diversos ejemplos de buenas prácticas sobre contratación de personas con discapacidad y sobre diseño de productos y servicios para que sean accesibles, actuaciones que deberían difundirse por todos los países de la Unión. Así, el Foro británico de empresarios sobre discapacidad actúa como una red de empresarios interesados por esta materia. En particular, los miembros del Foro facilitan ayuda a los que desean contratar a personas con discapacidad o mejorar el servicio que ofrecen a clientes con discapacidad.

\footnotetext{
${ }^{16}$ En el mismo periodo, el programa PROGRESS destinará 700 millones de euros a fomentar estudios de ámbito europeo que contribuyan a mejorar el conocimiento de las cuestiones sociales y laborales, formar profesionales en derecho y política, crear redes transfronterizas para estimular el aprendizaje y el intercambio de prácticas, y promover el conocimiento de las políticas de la UE. Por último, el Fondo Europeo de Adaptación a la Globalización dispone de 500 millones de euros anuales para facilitar ayuda personalizada a los trabajadores despedidos como consecuencia de la liberalización del comercio.
}

${ }^{17}$ Comunicación de la Comisión de 28 de noviembre de 2005 - Situación de las personas con discapacidad en la Unión Europea ampliada: el plan de acción europeo 2006-2007 [COM (2005) 604 final - No publicada en el Diario Oficial]. 
Además, en el ámbito comunitario, todos los años, el 3 de diciembre ${ }^{18}$, la Comisión organiza una conferencia para celebrar el Día Europeo de las Personas con Discapacidad, en estrecha colaboración con el Foro Europeo de Discapacidad comentado anteriormente. Los resultados y las conclusiones de estas conferencias han servido de referencia, en gran medida, para establecer las prioridades de las fases sucesivas del Plan de acción de la Unión, estando en vigor actualmente el Plan plurianual 2004-2010'19, que repasamos seguidamente.

\section{Igualdad de oportunidades para las personas con discapaci- dad: un plan de acción europeo (2004-2010)}

El fin del Plan de acción es integrar, desde 2004 y antes de 2010, la discapacidad en las políticas comunitarias (mainstreaming), gracias a la fijación de prioridades en todas las actividades de la Unión. El objetivo final de estas medidas es mejorar la integración económica y social de las personas con discapacidad. El mainstreaming requiere un diálogo constante con las autoridades públicas, los interlocutores sociales, el sector privado y las organizaciones no gubernamentales (ONG), que permitan anticiparse a cambios sociales y económicos, que afectan especialmente a los sectores más desfavorecidos en el mercado laboral, en un entorno marcado por el envejecimiento de la población europea.

El enfoque comunitario para ayudar a las personas con mayores dificultades está vinculado a los conceptos activos de integración, impulsando el reconocimiento y la protección de sus derechos, eliminando todo tipo de barreras físicas, técnicas, jurídicas, que no permitan una participación plena de estos ciudadanos europeos en el empleo. En cualquier caso, la efectividad de estas medidas pasa por su coherencia y complementariedad con los planes nacionales de acción establecidos por los propios Estados miembros, teniendo en cuenta que los criterios aplicados para determinar una discapacidad, no son idénticos en las diferentes legislaciones nacionales. Además, con el objeto de favorecer la movilidad de las personas con discapacidad, la Comisión se ha propuesto disminuir el número de prestaciones de

\footnotetext{
${ }^{18}$ Esta fecha coincide con la del Día Internacional de las Personas Discapacitadas, fijado por las Naciones Unidas en el año 1992.

19 Comunicación de la Comisión, de 30 de octubre de 2003, Igualdad de oportunidades para las personas con discapacidad: un plan de acción europeo [COM (2003) 650 final - no publicada en el Diario Oficial].
} 
invalidez que no son exportables de un Estado miembro a otro ${ }^{20}$ y ha establecido unos objetivos para los próximos años.

La Comisión ha propuesto tres objetivos operativos hasta 2010 que se completan y apoyan mutuamente:

A. Garantizar la plena aplicación de las Directivas 2000/78/CE y 2002/73/CE, anteriormente comentadas, relativas a la igualdad de trato en el empleo y la ocupación, y emprender un debate sobre la nueva estrategia de lucha contra la discriminación.

B. Conseguir la integración de la dimensión de la discapacidad en las políticas comunitarias afectadas en los procesos existentes (Estrategia Europea de Empleo, Estrategia Europea de Inclusión Social, etc.).

C. Mejorar la accesibilidad a los bienes, los servicios y el entorno construido.

Con el fin de alcanzar estos fines, la Comisión ha establecido un plan de acción plurianual continuo que abarcará un período que llegará hasta 2010. Un informe bienal, en el que se examinará la situación global de las personas con discapacidad, definirá y sustentará las sucesivas etapas de este programa. Asimismo, este informe servirá de base para la fijación de nuevas prioridades en el marco de las fases siguientes de la estrategia.

Respecto al primer objetivo (A), la Comisión ha establecido las siguientes orientaciones en una primera fase del Plan de Acción, en el ámbito de las personas con discapacidad: el acceso al empleo y el mantenimiento en la vida activa (A1); el aprendizaje permanente (A2); la utilización del potencial de las nuevas tecnologías (A3); y la accesibilidad del entorno público construido (A4).

\section{A. I.Acceso al empleo y mantenimiento en la vida activa}

En este punto, se pretende lograr una mayor sensibilización en los Estados miembros - a todos los niveles- sobre la dimensión laboral de la discapacidad, en el contexto de su responsabilidad social. Por lo que se insta a los gobiernos nacionales a intensificar los esfuerzos destinados a suprimir los obstáculos a la

\footnotetext{
${ }^{20}$ Modificación prevista del Reglamento (CEE) $n^{\circ}$ 1408/71 relativo a los regímenes de seguridad social.
} 
integración y la participación de los ciudadanos con discapacidad en el mercado de trabajo, en particular, y en la sociedad, en general. Los principales programas del FSE y la Iniciativa Comunitaria EQUAL ${ }^{21}$, han financiado una amplia serie de medidas destinadas a integrar a las personas con discapacidad en el mercado de trabajo y experimentan enfoques innovadores relativos a aspectos específicos de esta integración.

Asimismo, la Comisión ha actuado también en el marco de la política de la competencia, ya que como se apuntó anteriormente, en noviembre de 2002, se adoptó un reglamento relativo a las ayudas estatales para el empleo, en el que se autoriza a los Estados miembros a financiar hasta un $60 \%$ de los costes salariales anuales y de las cotizaciones de seguridad social cuando una empresa contrate a un trabajador con discapacidad. Asimismo, puede concederse una ayuda para compensar toda reducción de productividad o permitir una adaptación de las instalaciones.

En cuanto a la salud y la seguridad en el trabajo, la Directiva 89/654/CE relativa a las disposiciones mínimas de seguridad y de salud laboral, prevé que los lugares de trabajo deberán estar acondicionados teniendo en cuenta, en su caso, a los trabajadores minusválidos.

\section{A.2. Aprendizaje permanente}

Entre 2004-2006, se apostó firmemente por la potenciación de la educación y la formación de las personas con discapacidad, cuyas actuaciones se pretenden mantener hasta el final del programa. Por esa razón, se optó por el recurso a las tecnologías modernas de la información y la comunicación (TIC), que permite el aprendizaje en línea o elearning ${ }^{22}$, como soporte para eliminar los obstáculos a la educación, la formación y el aprendizaje permanente a que se enfrentan estos ciudadanos europeos. De este modo, la propuesta de programa elearning de la Comisión menciona expresamente las necesidades de las personas con discapacidad,

\footnotetext{
${ }^{21}$ La iniciativa comunitaria EQUAL está dirigida a promover nuevas prácticas de lucha contra todo tipo de discriminación y de desigualdad en el mercado de trabajo en un contexto de cooperación nacional y facilitar la inserción social y profesional de los solicitantes de asilo.

${ }^{22}$ Decisión N²318/2003/CE del Parlamento Europeo y del Consejo de 5 de diciembre de 2003 por la que se adopta un programa plurianual (2004-2006) para la integración efectiva de las tecnologías de la información y la comunicación (TIC) en los sistemas de educación y formación en Europa (programa elearning) [DO L 345 de 31.12.2003].
} 
al igual que el plan de acción relativo al aprendizaje de idiomas y la diversidad lingüística y el que tiene por objeto las capacidades y la movilidad.

Asimismo, se incorporó la información destinada a las personas con discapacidad en el sistema de información PLOTEUS ${ }^{23}$, un nuevo portal educativo comunitario para impulsar un sistema educativo, formativo y laboral europeo, integrado y sin fronteras, en el que se puede encontrar toda la información sobre las posibilidades de aprendizaje a lo largo de la vida en Europa.

Por otra parte, se prestó una atención especial a los proyectos de los programas de intercambio y becas Sócrates, Leonardo y Juventud en los que participan las personas con discapacidad.

\section{A.3. Explotar el potencial de las nuevas tecnologías}

La difusión y aprendizaje de las nuevas tecnologías brinda mayores opciones para que las personas con discapacidad puedan superar sus límites funcionales $y$, de este modo, evitar su exclusión de la tecnología digital.

Las medidas en el ámbito de accesibilidad, realizadas en el marco del plan de acción e-Europe ${ }^{24}$ (2002 y 2005), arrojaron resultados positivos respecto a la participación y la igualdad de los grupos más desfavorecidos a las grandes novedades en el ámbito de los servicios públicos en línea, la administración en línea (e-government), el aprendizaje electrónico (e-learning) y los servicios de salud en línea (e-health), así como para crear un entorno dinámico y accesible para el comercio electrónico (e-business) ${ }^{25}$.

En general, el objetivo era instaurar un entorno favorable a la inversión privada y a la creación de nuevos puestos de trabajo, impulsar la productividad, modernizar los servicios públicos y ofrecer a las personas discapacitadas la posibilidad de participar en la sociedad de la información mundial. En este ámbito, el plan

\footnotetext{
23 PLOTEUS tiene como objetivo ayudar a estudiantes, personas que buscan empleo, trabajadores, padres, orientadores y profesores a encontrar información sobre cómo estudiar en Europa.

${ }^{24}$ Comunicación de la Comisión al Consejo, al Parlamento Europeo, al Comité Económico y Social y al Comité de las Regiones e-Europe 2005: Una sociedad de la información para todos COM (2002) 263 final.

${ }^{25}$ Cfr. Cuenca, E. (2007)
} 
prioriza los siguientes aspectos: instauración de un diálogo internacional sobre las cuestiones e instrucciones relativas a la accesibilidad; inserción de criterios de accesibilidad para las TIC en los contratos públicos y los servicios públicos; estímulo del "diseño para todos", las tecnologías de asistencia y las normas de accesibilidad electrónica; y refuerzo del presupuesto destinado a investigación en materia de tecnologías sin obstáculos y "capacitadoras".

En definitiva, el presente plan de acción constituye una propuesta para que los Estados miembros asuman mayores compromisos en este ámbito y colaboren activamente junto al sector privado en el logro de los objetivos de eEurope, incorporando en este plan un lugar privilegiado a las personas con discapacidad.

\section{A.4. Accesibilidad del entorno público construido}

Para garantizar un acceso mejorado y efectivo de las personas con discapacidad al lugar de trabajo debe multiplicarse el diseño y la construcción de edificios que respeten el principio de diseño universal (o "diseño para todos"), con la presencia, asimismo, de infraestructuras culturales y de ocio accesibles ${ }^{26}$. En este sentido, el Consejo invitó a la Comisión, los Estados miembros y otros interesados a que intensificaran sus esfuerzos para facilitar la entrada de estos ciudadanos a las infraestructuras y actividades culturales y al turismo.

Por otra parte, en el Libro Blanco titulado "La política europea de transportes de cara al 2010: la hora de la verdad"27, la Comisión defiende que serán necesarias muchas medidas e instrumentos políticos para iniciar el proceso que desemboque en un sistema de transporte sostenible y sensible con las necesidades de las personas con discapacidad, avanzando en los siguientes aspectos: promoción de normas europeas en todos los ámbitos relativos al entorno construido, incluidas la planificación, el diseño, la construcción y la utilización de los edificios; fomento de la mejora de la enseñanza de la dimensión de la accesibilidad en las escuelas y para los profesionales; impulso a la integración de la accesibilidad en las políticas relativas a los contratos públicos y de la consideración de esta dimensión en el marco de la adjudicación de los Fondos Estructurales; y estímulo al desarrollo de estudios en materia de accesibilidad a los lugares e infraestructuras turísticos y a los transportes urbanos.

\footnotetext{
${ }^{26}$ Resolución del Consejo de 15 de julio de 2003 sobre el fomento del empleo y de la inclusión social de las personas con discapacidad Diario Oficial n ${ }^{\circ} \mathrm{C} 175$ de 24/07/2003 p. $0001-0002$.

${ }^{27}$ COM (2001) 370 final.
} 
La segunda fase de este plan de acción se desplegó, en un primer momento, en dos años (2006-2007) e insistía en la integración activa y la autonomía (derecho de las personas con discapacidad a ser destinatarias de medidas concebidas para garantizar su independencia, su integración social y profesional y su participación en la vida de la comunidad). En ella se proponían cuatro prioridades, con el control y seguimiento del grupo interservicios de la Comisión Europea, que se ocupa de las cuestiones relativas a las personas con discapacidad: fomentar la actividad profesional; promover el acceso a una asistencia y a servicios de cuidados de calidad; promover el acceso de todos a los bienes y los servicios; e incrementar la capacidad de recogida y análisis de la Unión.

Por su parte, el Grupo europeo de alto nivel responsable de los temas relacionados con las personas con discapacidad (grupo de expertos presidido por la Comisión y que reúne a especialistas de los Estados miembros), se encarga de desarrollar las sinergias entre las políticas nacionales y las comunitarias.

Finalmente, hay que destacar que la Comisión ha realizado una primera evaluación del plan de acción en 2008 , con un especial interés por seguir los esfuerzos realizados para promover la igualdad de oportunidades de las personas con discapacidad. Se presentarán las contribuciones específicas de los Estados miembros en las que se muestren sus realizaciones en el ámbito de las personas con discapacidad, sobre la base, entre otras disposiciones, de las principales iniciativas comunitarias comentadas en este trabajo, impulsadas por las diferentes instituciones: Comisión Europea (A), el Consejo de Europa (B) y el Parlamento Europeo (C):

\section{A) Comisión Europea}

Año 2000. Hacia una Europa sin barreras para las personas con discapacidad.

Año 2001. Año Europeo de las personas con discapacidad 2003.

Año 2003. Plan de acción europeo para la igualdad de oportunidades para las personas con discapacidad (2004-2010).

Año 2003. Promoción y protección de los derechos y la dignidad de las personas con discapacidad a escala internacional.

Año 2004. Libro Verde. Igualdad y no discriminación en la Unión ampliada.

Año 2005. Situación de las personas con discapacidad en la Unión Europea ampliada: el plan de acción europeo 2006-2007. 
B) El Consejo de Europa

Año 1998. Recomendación 98/376/CE del Consejo, de 4 de junio de 1998, sobre la creación de una tarjeta de estacionamiento para personas con discapacidad.

Año 2000. Directiva 2000/78/CE del Consejo, de 27 de noviembre de 2000, relativa al establecimiento de un marco general para la igualdad de trato en el empleo y la ocupación.

Año 2001. Decisión 2001/903/CE del Consejo, de 3 de diciembre de 2001, sobre el Año Europeo de las personas con discapacidad 2003.

Año 2002. Directiva 2002/73/CE del Consejo, de 23 de septiembre de 2002, relativa a la aplicación del principio de igualdad de trato en lo que se refiere al acceso al empleo, a la formación y a la promoción profesionales, y a las condiciones de trabajo.

\section{C) El Parlamento Europeo}

Año 2005. Actualización del "Código de buenas prácticas", sobre el empleo de personas con discapacidad en el Parlamento Europeo.

Año 2006. Informe del Parlamento sobre la necesidad de mejorar el acceso de las personas discapacitadas al transporte público y a Internet.

\section{Un instrumento jurídicamente vinculante de Naciones Unidas en materia de discapacidad a escala internacional.}

La Unión pretende potenciar en los próximos años su cooperación con organizaciones como las Naciones Unidas, la Agencia europea para la seguridad y la salud en el trabajo, organizaciones europeas de normalización tales como el Centro Europeo de Normalización (CEN), la Agencia europea de la educación adaptada a necesidades especiales y la Fundación para la mejora de las condiciones de vida, con el objeto de llevar a cabo medidas que favorezcan a las personas con discapacidad, aprovechando, a su vez, su experiencia y el trabajo de sus grupos de reflexión. En particular, la Comisión apoya y participa activamente en la instauración de un instrumento jurídicamente vinculante de Naciones Unidas para 
proteger y promover los derechos y la dignidad de las personas con discapacidad a escala internacional ${ }^{28}$.

Aunque este principio está establecido por los instrumentos internacionales de protección de los derechos humanos, y se aplica, generalmente, a las personas con discapacidad, estas últimas no disfrutan satisfactoriamente en determinadas ocasiones de sus derechos. Por todo ello, la Comisión Europea secunda a las Naciones Unidas en su objetivo de garantizar que las personas con discapacidad no sean objeto de discriminación y puedan acceder a sus derechos y ejercerlos al igual que las demás personas.

En esencia, se pretende adoptar un instrumento jurídicamente vinculante en el marco de las Naciones Unidas relativo a las personas con discapacidad para poner de manifiesto que estos ciudadanos tienen los mismos derechos que el resto de la humanidad, completando, al mismo tiempo, la normativa existente en materia de protección de los derechos humanos. Desde 2002, un Comité especial, creado en el marco de las Naciones Unidas a raíz de la Resolución 56/168, examina las propuestas a fin de elaborar un convenio internacional global e integrado para la promoción y la protección de los derechos y de la dignidad de las personas con discapacidad. El instrumento deberá proteger contra toda discriminación en el acceso y el disfrute de los derechos humanos.

La elaboración de un instrumento jurídicamente vinculante permitiría reforzar la coherencia entre la acción internacional y la acción comunitaria, de lo que se encargaría la Comisión. Con estos objetivos, esta última remitió una Recomendación al Consejo para solicitar un mandato de negociación en nombre de la Comunidad Europea relativo a la elaboración en el marco de las Naciones Unidas de un convenio internacional global e integrado para la promoción y la protección de los derechos y la dignidad de las personas con discapacidad ${ }^{29}$.

${ }^{28}$ Comunicación de la Comisión al Consejo y al Parlamento Europeo, de 24 de enero de 2003, "Hacia un instrumento jurídicamente vinculante de las Naciones Unidas para promover y proteger los derechos y la dignidad de las personas con discapacidad" [COM (2003) 16 final - no publicada en el Diario Oficial].

${ }^{29}$ Recomendación de la Comisión al Consejo, de 31 de enero de 2003, para que se autorice a la Comisión a participar en las negociaciones dirigidas a la elaboración de un instrumento internacional jurídicamente vinculante para promover y proteger los derechos y la dignidad de las personas con discapacidad [SEC (2003) 116 final - no publicada en el Diario Oficial]. 
La Comisión estima que la experiencia comunitaria en las actuaciones en el ámbito de las personas con discapacidad puede suponer un apoyo para el resto del mundo, sobre la base de los siguientes aspectos: consolidar la cooperación con los países y entre ellos; crear un grupo de alto nivel compuesto por representantes de los Estados miembros para las cuestiones relacionadas con la discapacidad; entablar un diálogo social centrado en los problemas relacionados; seguir apoyando a las organizaciones no gubernamentales activas en el ámbito de estos ciudadanos a fin de promover la cooperación europea; integrar la cuestión de la discapacidad en la formulación de propuestas de políticas comunitarias; reforzar las medidas destinadas a la prevención del desempleo de larga duración y a la incorporación de las personas con algún tipo de minusvalía en la vida profesional, en el marco de la estrategia europea para el empleo; evaluar el alcance y la repercusión de las acciones relativas a los grupos más desfavorecidos, particularmente en el contexto de la lucha contra la exclusión social.

Con este objetivo, el Consejo insta a la Comisión a incorporar la dimensión "igualdad de oportunidades de las personas con discapacidad" en todas las acciones comunitarias que lo permitan ${ }^{30}$. Al mismo tiempo, invita a los Estados miembros a que pongan especial énfasis en fomentar las oportunidades de empleo de las personas minusválidas y adopten las medidas preventivas que fomenten su integración en el mercado laboral; utilicen plenamente las posibilidades existentes y futuras de los Fondos Estructurales europeos, especialmente las del Fondo Social Europeo; y presten especial atención a las posibilidades que ofrece el desarrollo de la sociedad de la información.

Como consecuencia, en el marco de la estrategia internacional de la ONU, en el seno de la Unión Europea, las disposiciones nacionales consideradas discriminatorias respecto a las personas con discapacidad deberán suprimirse o declararse nulas y sin efecto. En particular, los Estados miembros adoptarán sanciones en caso de violación del principio de igualdad de trato. Asimismo, comunicarán a la Comisión cualquier información necesaria para la redacción de un informe dirigido al Parlamento Europeo y al Consejo sobre la aplicación de estas actuaciones.

Y, como culminación de todas estas actuaciones, la Asamblea General de la ONU, en su $76^{a}$ sesión plenaria del 13 de diciembre de 2006, aprobó la Resolución 61/06, relativa a la Convención sobre los derechos de las personas con discapacidad.

\footnotetext{
${ }^{30}$ Resolución del Consejo y de los representantes de los Gobiernos de los Estados miembros reunidos en el seno del Consejo, de 17 de junio de 1999, relativa a la igualdad de oportunidades laborales de las personas con discapacidad [Diario Oficial C 186 de 2.7.1999].
} 


\section{Algunas consideraciones finales}

En la estrategia a largo plazo de la Unión para la integración laboral de las personas discapacitadas, se pretende alcanzar mejoras reales en las perspectivas de empleo, la accesibilidad y la vida independiente, de acuerdo con su visión de que la discapacidad es una cuestión de derecho y no de discreción. En particular, en los países de la Unión se ha pasado de un sistema tradicional en el que las políticas implementadas hacia el colectivo tenían un corte social y asistencial hasta otro en el que la política de empleo se convierte en esencial como factor de integración de los discapacitados. Así, la mejora de la situación laboral de estas personas tendría un efecto positivo, no sólo para estas últimas, sino también para el conjunto de la sociedad europea.

En este sentido, hay que resaltar los crecientes esfuerzos de las instituciones europeas en la lucha contra cualquier tipo de discriminación, especialmente desde la entrada en vigor del Tratado de Ámsterdam, en lo que respecta a la integración de las personas discapacitadas, en el marco de la Política Social Europea y en línea con las medidas de Naciones Unidas y con la reglamentación de los Estados miembros. No obstante, se trata, en gran medida, de un objetivo que debe lograrse y todavía no de una realidad, de tal modo que la estrategia comunitaria en la dimensión de las personas discapacitadas en general, y el proceso de mainstreaming, en particular, quedará más limitado en su eficacia si no se dispone de una mayor financiación dedicada a este objetivo.

Con todo, y aunque la política sobre discapacidad aún es competencia nacional, las diferentes iniciativas de la Unión, que hemos descrito en nuestro trabajo, han influido positivamente en las políticas de los Estados miembros sobre las personas con discapacidad. Sin embargo, el análisis de la situación de las personas discapacitadas en Europa arroja aún un resultado negativo, especialmente en términos de altas tasas de desempleo, si bien la situación varía en función de los países. Por consiguiente, la estrategia futura sobre discapacidad deberá potenciarse en mayor medida en todos los aspectos y tener en cuenta una complementariedad adecuada entre las políticas nacionales y las comunitarias, junto con las competencias cada vez mayores de los entes regionales y locales.

En particular, en el área de la igualdad de derechos y oportunidades en el acceso al empleo, la Unión ofrece apoyo financiero a través de múltiples instrumentos, que se han descrito en los puntos anteriores, si bien se debería mejorar la integración de las necesidades de las personas con discapacidad en la Estrategia Europea de Empleo y las medidas del Fondo Social Europeo. Paralelamente, sería necesario 
potenciar también otro tipo de acciones: diálogo social, desarrollo de un marco legal armonizado a escala comunitaria, mejora de la coordinación de los sistemas de empleo nacionales y políticas de inclusión con objetivos cuantificados, entre otros. Es este contexto, los ciudadanos individuales, la sociedad civil y las ONG deben trabajar en la concienciación sobre estos problemas y asumir un papel más activo en la búsqueda de soluciones específicas.

Asimismo, sería conveniente disponer de un mayor número de medidas legalmente vinculantes en relación a la discapacidad, con el fin de evitar cualquier tipo de discriminación en los Estados miembros, ya que el Derecho Comunitario es la piedra angular de una sociedad europea de inclusión, que proporcione nuevas oportunidades e impulse cambios relevantes en este campo. Por este motivo, la legislación europea anti-discriminación se debería aplicar en todas partes con el mismo grado de eficacia, puesto que los Programas de Ayudas a las personas discapacitadas difieren aún de unos países a otros, dependiendo de aspectos políticos, culturales, económicos, sociales, etc. Por lo que es necesario que la discapacidad tenga una presencia cada vez mayor en las políticas comunitarias, con una armonización en unas bases comunes en las legislaciones nacionales.

Las actividades para beneficiar a las personas con discapacidad deben ser una práctica regular en todos los países e instituciones de la Unión Europea. En este sentido, se considera conducta inapropiada, ignorar o rechazar las necesidades de las personas con discapacidad en el diseño y la puesta en práctica de la política social y de las operaciones ordinarias de las instituciones comunitarias.

En cualquier caso, y en el marco del cumplimiento de las disposiciones derivadas del Derecho Interno de la Unión y de la Convención Internacional de Naciones Unidas, los Estados miembros están obligados a seguir introduciendo medidas destinadas a promover los derechos de las personas con discapacidad. Además, deben profundizar en sus acciones, con un desarrollo cada vez mayor de la legislación antidiscriminatoria, al mismo tiempo que tienen que ampliar la prestación de servicios, los bienes y las infraestructuras accesibles a las personas con discapacidad. Además, los avances en la integración de las personas con discapacidad no deberían limitarse a los ciudadanos que viven en la Unión Europea, sino que debería englobar a las de los países candidatos, pero también a los discapacitados de los países en desarrollo, que podrán beneficiarse de la mayor visibilidad en las políticas comunitarias de estas actuaciones.

Para garantizar la inclusión activa de personas con discapacidad en todos los campos de la vida, debemos apoyar la adopción de una legislación completa, 
que refuerce la protección frente a la discriminación en el mercado de trabajo, y extenderla a todos los demás aspectos de la vida, por lo que se debe garantizar la plena aplicación de la Directiva relativa a la igualdad de trato en el empleo y la ocupación. En este marco, la estrategia comunitaria actual en este ámbito gira en torno a tres pilares: 1) legislación y medidas contra la discriminación, que dan acceso a los derechos individuales; 2) eliminación de las barreras físicas que impiden que las personas con discapacidad puedan desarrollar su potencial; y 3) incorporación de los aspectos relacionados con la discapacidad en la amplia gama de políticas comunitarias para facilitar la inclusión activa de estas personas.

Hay que tener en cuenta que la administración de los sistemas de protección e integración social de las personas discapacitadas, su financiación y la oferta de asistencia, de acuerdo las necesidades de la población, son competencia de los gobiernos nacionales. No obstante, esta atribución se desarrolla cada vez más en un contexto global, en el que participan múltiples políticas comunitarias, lo cual recomienda una cooperación cada vez mayor en este ámbito en el seno de la Unión, tanto desde una óptica territorial como institucional.

Por último, consideramos importante para mejorar la inclusión activa de las personas discapacitadas en la sociedad y la economía europea promover un debate abierto y continuo entre los Estados miembros sobre la problemática de la integración laboral de este colectivo, con la participación de las instituciones comunitarias, los gobiernos nacionales, las autoridades regionales y locales, los agentes sociales, expertos universitarios, asociaciones de personas discapacitadas, las ONG, etc. 


\section{Bibliografía}

Asambiea General de Naciones Unidas (2008) Sexagésimo segundo período de sesiones; Tema 70 e) del programa: Promoción y protección de los derechos humanos: Convención sobre los derechos de las personas con discapacidad, 22 de enero de 2008 (A/62/654).

CASADO, D. (2006) "Respuestas a la dependencia funcional y agentes", Revista Española del Tercer Sector, $\mathrm{n}^{\circ}$ 6, pp. 1-21.

Comisión Europea (2005a) Comunicación de la Comisión de 28 de noviembre de 2005 Situación de las personas con discapacidad en la Unión Europea ampliada: el plan de acción europeo 2006-2007, [COM (2005) 604 final - No publicada en el Diario Oficial].

Comisión Europea (2005b) Comunicación de la Comisión al Consejo, al Parlamento Europeo, al Comité Económico y Social Europeo y al Comité de las Regiones, de 1 de junio de 2005, "Estrategia marco contra la discriminación y por la igualdad de oportunidades para todos" [COM (2005) 224 - no publicada en el Diario Oficial].

COMISIÓN EUROPEA (2004a) Libro Verde. Igualdad y no discriminación en la Unión Europea ampliada [COM (2004) 379 final - no publicado en el Diario Oficial].

Comisión Europea (2004b) Comunicación de la Comisión, de 20 de abril de 2004 Modernizar la protección social para el desarrollo de una asistencia sanitaria y una asistencia de larga duración de calidad, accesibles y duraderas: apoyo a las estrategias nacionales a través del "método abierto de coordinación", [COM (2004) 304 final - no publicada en el Diario Oficial].

Comisión Europea (2003a) Comunicación de la Comisión, de 30 de octubre de 2003, Igualdad de oportunidades para las personas con discapacidad: un plan de acción europeo, [COM (2003) 650 final - no publicada en el Diario Oficial].

Comisión Europea (2003b) Comunicación de la Comisión al Consejo y al Parlamento Europeo, de 24 de enero de 2003, "Hacia un instrumento jurídicamente vinculante de las Naciones Unidas para promover y proteger los derechos y la dignidad de las personas con discapacidad", [COM (2003) 16 final - no publicada en el Diario Oficial]. 
Comisión Europea (2002) Comunicación de la Comisión al Consejo, al Parlamento Europeo, al Comité Económico y Social y al Comité de las Regiones eEurope 2005: Una sociedad de la información para todos, COM (2002) 263 final.

Comisión EuROPEA (2001) Comunicación de la Comisión al Consejo: El futuro de la asistencia sanitaria y de la atención a las personas mayores: garantizar la accesibilidad, la calidad y la sostenibilidad financiera, (COM (2001) 723 final).

Comisión EuRopea (2000) Directiva 2000/78/CE del Consejo, de 27 de noviembre de 2000, relativa al establecimiento de un marco general para la igualdad de trato en el empleo y la ocupación. DO L 303 de 2.12.2000.

Comisión EUROPEA (1999) Comunicación de la Comisión sobre algunas medidas comunitarias de lucha contra la discriminación (COM (1999) 564 final).

COMISIÓN EUROPEAMISSOC (varios años) La protection sociale dans les États membres de l'Union européenne. Commission Européenne.

Consejo de Europa (2003a) Resolución del Consejo y de los representantes de los Gobiernos de los Estados miembros reunidos en el seno del Consejo, de 15 de julio de 2003, sobre el fomento del empleo y de la inclusión social de las personas con discapacidad, [Diario Oficial C 175 de 24.7.2003].

Consejo de Europa (2003b) Decisión № 2318/2003/CE del Parlamento Europeo y del Consejo de 5 de diciembre de 2003, por la que se adopta un programa plurianual (2004-2006) para la integración efectiva de las tecnologías de la información y la comunicación (TIC) en los sistemas de educación y formación en Europa (programa elearning), [DO L 345 de 31.12.2003].

Consejo de Europa (2001) Decisión 2001/903/CE del Consejo, de 3 de diciembre de 2001, sobre el Año Europeo de las personas con discapacidad 2003, [Diario Oficial L 335 de 19.2.2001].

Consejo de Europa (2000) Decisión del Consejo, de 27 de noviembre de 2000, por la que se establece un programa de acción comunitario para luchar contra la discriminación (2001-2006, (DO L 303 de 2.12.2000).

Consejo de Europa (1999) Resolución del Consejo y de los representantes de los Gobiernos de los Estados miembros reunidos en el seno del Consejo, de 17 de junio de 1999, relativa a la igualdad de oportunidades laborales de las personas con minusvalías, [Diario Oficial C 186 de 2.7.1999]. 
Consejo de EUROPA (1996) Resolución del Consejo y de los representantes de los gobiernos de los Estados miembros reunidos en el seno del Consejo de 20 de diciembre de 1996, sobre la igualdad de oportunidades para las personas con minusvalías [Diario Oficial C 12 de 13.1.1997].

CuencA, E. (2007) Economía de la Unión Europea, Madrid, Pearson.

LavíN, M. (2006) "Modelos de protección de la dependencia en la Unión Europea y factores de convergencia", Revista andaluza de relaciones laborales, $\mathrm{n}^{\circ} 18$, 2006, pp. 17-37.

PéreZ, V. (2004) "Prestaciones de dependencia: situación comparada en la Unión Europea", Revista del Ministerio de Trabajo y Asuntos Sociales, n 47, pp. 295-324.

SAntos, M. del P. y Millán-Vázquez, G. (2008) "Discapacidad y accesibilidad: la lucha por la plena integración", Revista de Fomento Social, n² 250, abril-junio, pp. 275-288

ZoLKOWSKA, T. (2002) “Un resumen de las políticas de la Unión Europea relacionadas con la discapacidad", Journal, Disability Studies Quarterly, n 4, pp. 25-41. 